\title{
Do You Know Where Your Research is Being Used? An Exploration of Scientific Literature Using Natural Language Processing
}

\author{
Theodore J. LaGrow*, Computer and Information Science and Mathematics \\ Jacob Bieker**, Physics and Computer and Information Science \\ Boyana Norris ${ }^{* * *}$, Computer and Information Science
}

\begin{abstract}
In a complex and dynamic field, such as computer science, it is of interest to understand what software resources are available and the usage and purpose of these resources. We demonstrate the feasibility of automatically identifying resource names from scientific literature in arXiv's database and show that the generated data can be used for exploration of software and topics. While scholarly literature surveys can provide some insights on what is being used by researchers, large-scale computer-based approaches to identify methods and technology from primary literature is needed to enable systematic cataloguing. Further, these approaches will facilitate the monitoring of usage in a more effective method. We developed a software tool using Natural Language Processing to determine if articles relate to the technology and methods of question. We then evaluated a trend of technology and methods used in each specific area of science. As we continue to expand this software, we will also analyze the researchers' sentiment about the technology and methods to quantify funded research.
\end{abstract}

\footnotetext{
*Theodore J. LaGrow is a senior majoring in both Computer and Information Science and Mathematics and minoring in both Physics and Theatre Arts. Theodore's research interests include Biomedical Engineering, Natural Language Processing, Embedded Systems, Compound Hawkes Processes, and Shakespeare. Please direct correspondence to tlagrow@uoregon.edu.

** Jacob Bieker is a junior majoring in both Physics and Computer and Information Science. Jacob's research interests include Galaxy Evolution, Natural Language Processing, Particle Physics, High-Powered Dynamic Systems, Telescopes and Topographical Modeling.

***Boyana Norris is an Associate Professor in the Computer and Information Science Department at the University of Oregon. Boyana is the head of the High-Performance Computing Laboratory. Boyana graduated from the University of Illinois at Urbana-Champaign in 2000 with a PhD in Computer Science. Boyana's research interests include: complex high-performance applications for rapidly evolving parallel architectures, compiler techniques for source code analysis, embeddable domain-specific languages for code generation, and quality of service infrastructure for scientific software.
} 


\section{INTRODUCTION}

With expanding databases of scientific articles, there is rapidly growing access to publications on specific scientific topics. Hucka and Grahams (2016) suggest in their article "Software search is not a science, even among scientists," that the best approaches when searching for software ready to use are: "(i) search the Web with general-purpose search engines, (ii) ask colleagues, (iii) look in the scientific literature.” These dated technology search methods can be painstaking and arduous. These laborious searches cannot cover the amount of articles a program can parse through. We aimed to determine if there was a method to finding trends of technology usage by analyzing large data from these databases.

Recently, linguistic machine learning has been implemented to draw inference across large data sets (Bird et al., 2009). Scientific databases can be incorporated into large sets of collections from a given number of articles by using various methods for text extraction and filtering. Linguistic machine learning can be used to understand connections between documents within a given dataset. We decided to use natural language processing to explore and infer the prevalent technologies and methods used in various disciplines of science.

\section{NATURAL LANGUAGE PROCESSING OVERVIEW}

Bird et al. (2009) describe natural language processing (NLP) as the ability of a computer program to understand human speech as it is spoken. Natural language processing is a field of artificial intelligence and computational linguistics concerned with the interactions between computers and natural languages. Modern NLP is based on machine learning, especially statistical machine learning. The programing paradigm of machine learning differs from most prior attempts at language processing. Up to the 1980s, most NLP systems were based on complex sets of hand-written rules (Jones, 2001). Starting in the late 1980s, however, there was a revolution in NLP with the introduction of machine learning algorithms for language processing. This was due to the steady increase in computational power over time (Jones, 2001). Machine learning calls for using general learning algorithms, often grounded in statistical inference. The main idea is to automatically learn such rules through the analysis of large corpora of typical realworld examples. A corpus is a set of documents (or sometimes, individual sentences or strings) that have been hand-annotated with the correct values to be learned. The accuracy of the analysis can vary depending on the format of the data. The cleaner the data and corpus, the better the desired output.

\section{METHODS}

To obtain the data, we first parsed through arXiv.org search results for our topics of interest. arXiv.org is a major online hub where researchers pre-publish their articles while their papers get peer-reviewed. The four topics we considered were galaxy evolution, Hawkes processes, T-cell receptor genomes, and natural language processing itself. We downloaded PDF articles, then sorted them, extracting text using PDFminer (Shinyama, 2014) and Python (van Rossum, 1991). We decided to extract only the first 100 articles from the topic searches because of the limited computing capabilities available: Windows 10 desktop (specification: i7 core processor and 32GB RAM); a Windows 10 laptop (specification: i5 core processor and 6GB RAM); and a MacBook Pro 
(specification: i7 core processor and 8GB RAM). Once we converted the PDFs to text, we applied filters to the text to remove non-alphanumeric characters and any lines that were less than seven characters. Once the documents were cleaned in this manner, we used the Natural Language Toolkit ("Natural Language Toolkit," 2016) to parse the text, giving us the parts of speech of each word, a frequency distribution of n-grams containing predefined interesting words, and lists of words similar to the user-defined interesting words. N-grams take an interesting word and use it as a center point in the string of a given length $n$. Table 1 contains the interesting words we found that generated an output of comprehensive results. This optimization came after testing a list of words used when describing data.

Table 1: Interesting words used for n-grams

\section{Dictionary of Interesting Words}

simulation, software, code, analysis, using, program, analyzed, scripted, automated, description, implements, function, modifies, operated, pipeline, helps, allows, manipulate, processed

We decided to use n-grams of length 15 because the average length of a sentence is 6-7 words giving us roughly the sentence on either side of the interesting word. Once that was done, we traversed the collection of n-grams, only taking the noun phrases from the n-grams and counting the occurrences of each noun phrase. The counted noun phrases became the basis for the generated word clouds, which visualize the hierarchical significance of the word to the corpus of data related to the discipline being examined.

\section{RESULTS}

We found that each data set produced a variety of similar words. A few similar words included function, method, and analysis. These words had relatively high frequencies compared to the more unique words related to the data sets. We suspect that because these words are in our interesting words dictionary, they typically occur close to the other interesting words in our corpus. This would affect the frequency of the higher words due to commonality of the interesting dictionary words. Interesting results we found included: Gadget (a galaxy imaging technology), Velvet (an assembly program), and morphological (a method dealing with the structure of things). Both the technologies and the method extracted pertain heavily to each field: Hawkes processes, galaxy evolution, T-cell receptor genome, and natural language processing. We did not know the technology Gadget before we searched the database. This output signifies that our method of extraction will produce additional technology that may not be known to the user.

\subsection{OUTPUT FREQUENCIES}

Our first target was analyzing publications on Hawkes processes. Table 2 displays the top thirty noun frequencies as a result of our analysis. Figure 1 shows these words sized by the frequency of words within the document set. 
Table 2: Top 30 words and frequencies generated with search phrase: Hawkes process

\begin{tabular}{|c|c|}
\hline Word & Number of Occurrences \\
\hline Hawkes & 815 \\
\hline Rate Function & 349 \\
\hline Large Deviation Principle & 113 \\
\hline Lemma & 109 \\
\hline Exciting Function & 107 \\
\hline Point Processes & 99 \\
\hline $\mathrm{Eq}$ & 95 \\
\hline Theorem & 90 \\
\hline Poisson & 82 \\
\hline Fig & 78 \\
\hline Residual Analysis & 78 \\
\hline Hawking & 74 \\
\hline $\mathrm{Ix}$ & 70 \\
\hline Black Hole & 67 \\
\hline Intensity Function & 58 \\
\hline Correlation Function & 54 \\
\hline Conditional Intensity Function & 54 \\
\hline Contrast Function & 51 \\
\hline Excitement Function & 51 \\
\hline Consider & 49 \\
\hline Genome Analysis & 42 \\
\hline Numerical Simulations & 44 \\
\hline Simulation Study & 44 \\
\hline Morphological & 42 \\
\hline Partition Function & 42 \\
\hline Exponential Function & 40 \\
\hline Distribution Function & 39 \\
\hline Cost Function & 39 \\
\hline Kernel Function & 38 \\
\hline Wienerhopf & 38 \\
\hline Fourier & 37 \\
\hline
\end{tabular}




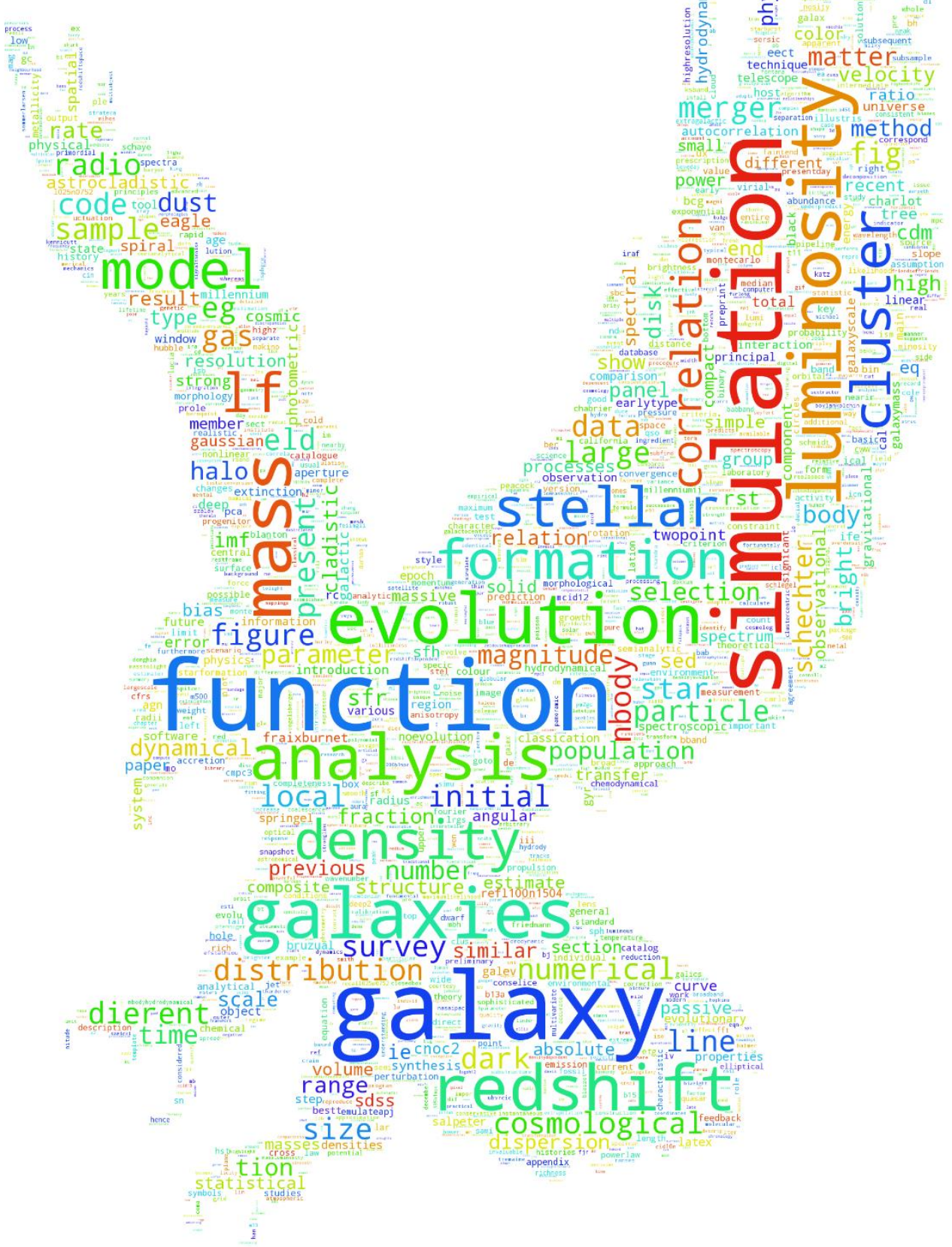

Figure 1: Output distribution word cloud of the search phrase: Hawkes process 
Our second target was analyzing publications on galaxy evolution. Table 3 displays the top thirty noun frequencies as a result of our analysis. Figure 2 shows these words sized by the frequency of words within the document set.

Table 3: Top 30 words and frequencies generated with search phrase: galaxy evolution

\begin{tabular}{|c|c|}
\hline Word & Number of Occurrences \\
\hline Luminosity Function & 332 \\
\hline N-body & 145 \\
\hline Fig & 128 \\
\hline Schechter & 101 \\
\hline Exciting Function & 107 \\
\hline Point Processes & 99 \\
\hline $\mathrm{Eq}$ & 95 \\
\hline Galaxy Luminosity Function & 72 \\
\hline Galaxy Evolution & 71 \\
\hline Galaxy Formation & 70 \\
\hline $\mathrm{CDM}$ & 67 \\
\hline Phylogenetic Analysis & 65 \\
\hline Body Simulations & 63 \\
\hline Numerical Simulations & 60 \\
\hline Initial Mass Function & 54 \\
\hline Cosmological Simulations & 53 \\
\hline Astrocladistics & 52 \\
\hline Mass Function & 49 \\
\hline Stellar Mass & 45 \\
\hline Transfer & 45 \\
\hline Eagle & 45 \\
\hline Local Density & 39 \\
\hline Compact Galaxies & 39 \\
\hline Gaussian & 37 \\
\hline Cladistic Analysis & 36 \\
\hline Gadget-3 & 36 \\
\hline Radio Galaxy Luminosity Function & 36 \\
\hline Star Formation & 36 \\
\hline Cluster Galaxies & 33 \\
\hline Correlation Function & 33 \\
\hline Bright End & 33 \\
\hline
\end{tabular}




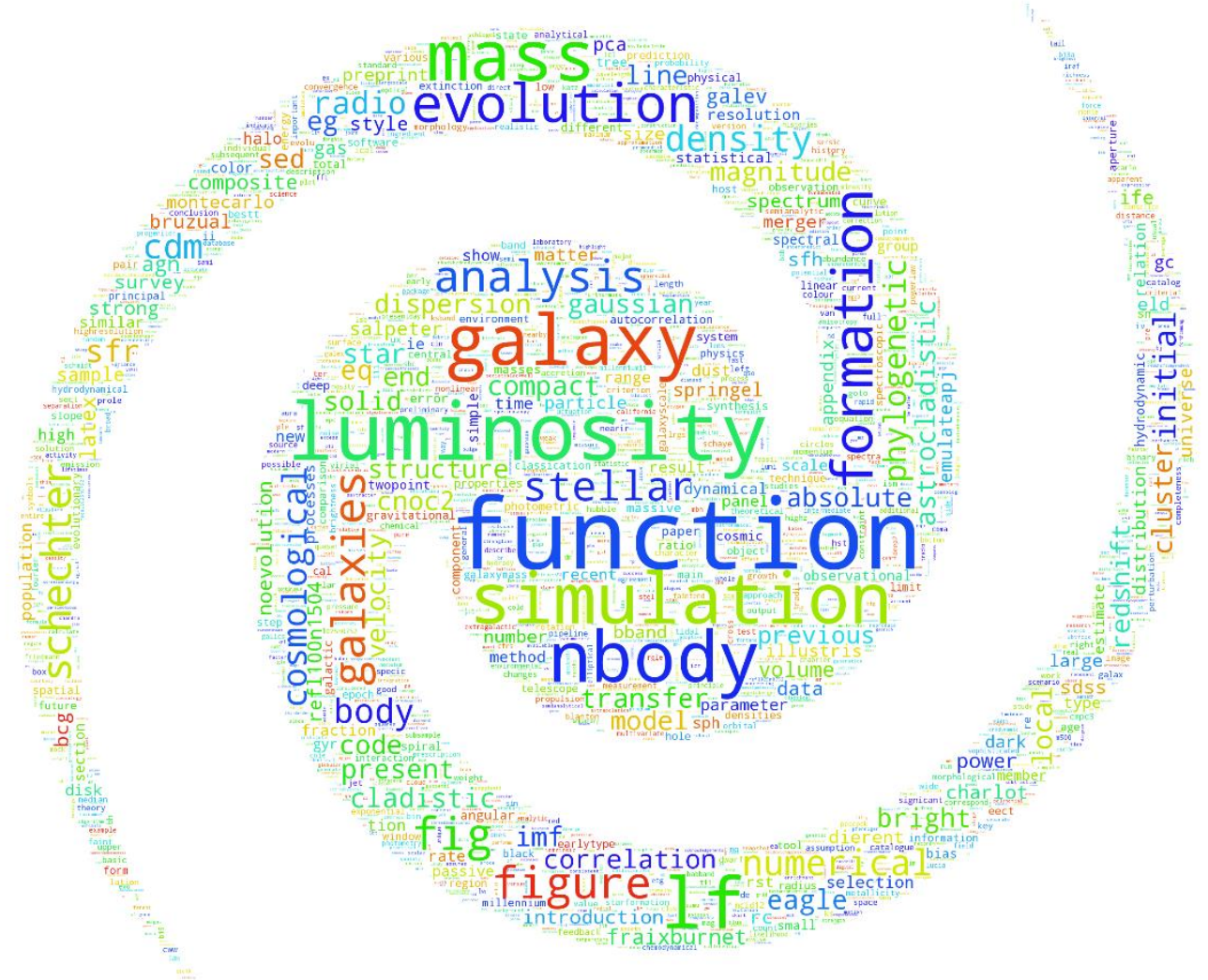

Figure 2: Output distribution word cloud of the search phrase: galaxy evolution 
Our third target was analyzing publications on T-cell receptor genome. Table 4 displays the top thirty noun frequencies as a result of our analysis. Figure 3 shows these words sized by the frequency of words within the document set.

Table 4: Top 30 words and frequencies generated with search phrase: T-cell receptor genome

\begin{tabular}{|c|c|}
\hline Word & Number of Occurrences \\
\hline Monte Carlo & 244 \\
\hline $\mathrm{Eq}$ & 244 \\
\hline Fig & 119 \\
\hline TCR & 82 \\
\hline DNA & 71 \\
\hline RNA & 68 \\
\hline SNPS & 59 \\
\hline Chipseq & 59 \\
\hline Numerical Simulations & 58 \\
\hline Partition Function & 54 \\
\hline Ligand Concentration & 53 \\
\hline Methods & 52 \\
\hline Correlation Function & 52 \\
\hline MC & 50 \\
\hline Gillespie & 46 \\
\hline RNAseq & 44 \\
\hline Microarray Analysis & 43 \\
\hline Maximum Likelihood & 41 \\
\hline Bayesian & 39 \\
\hline Simulation Study & 39 \\
\hline Velvet & 39 \\
\hline Data Analysis & 38 \\
\hline SNP & 37 \\
\hline Stochastic Simulation & 36 \\
\hline Cluster Size & 36 \\
\hline Covariance Function & 35 \\
\hline Dierent Values & 30 \\
\hline Greens & 28 \\
\hline Phylogenetic Analysis & 27 \\
\hline Quantitative Analysis & 27 \\
\hline
\end{tabular}




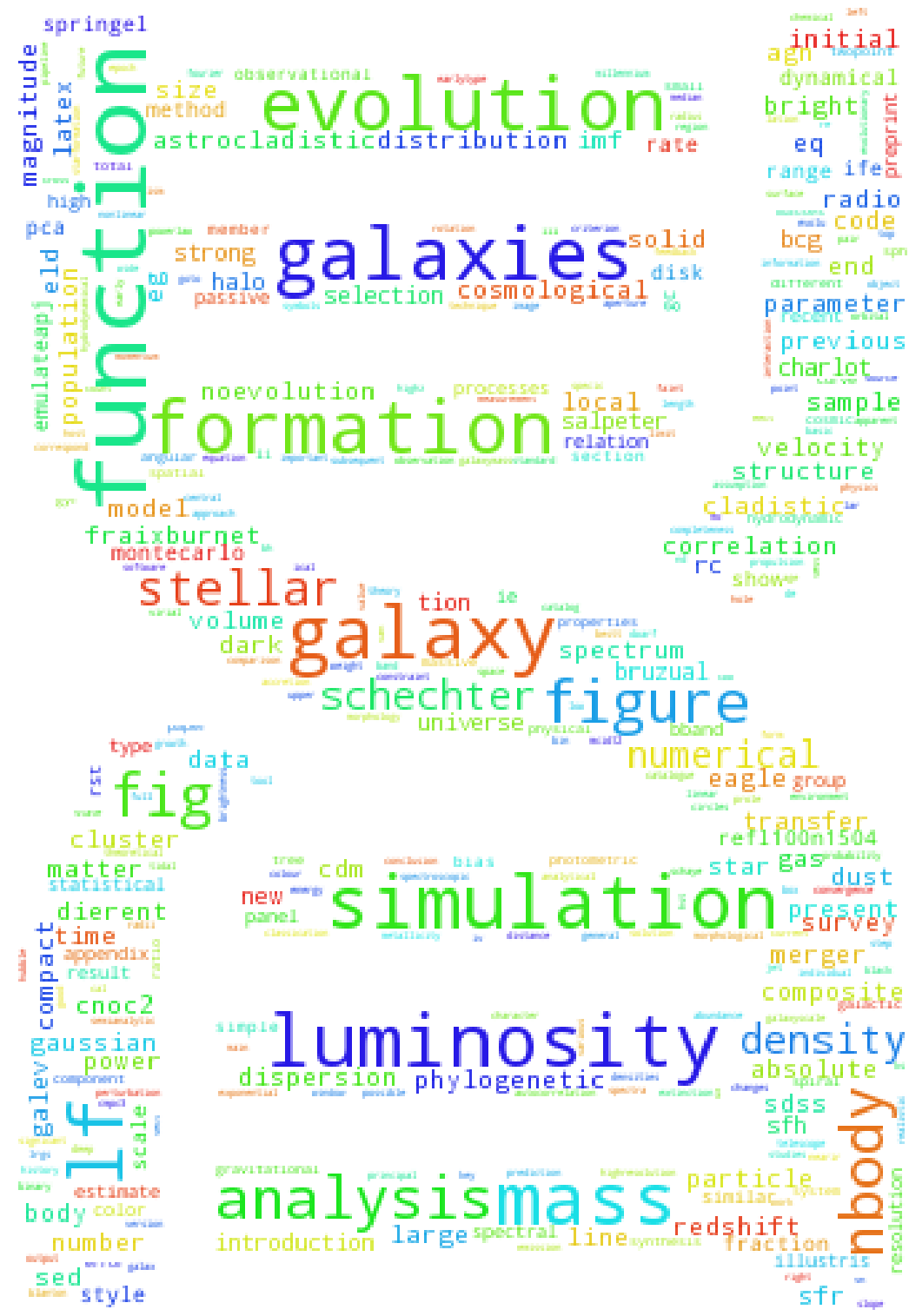

Figure 3: Output distribution word cloud of the search phrase: T-cell receptor genome 
Our fourth target was analyzing publications on Natural Language Processing. Table 5 displays the top thirty noun frequencies as a result of our analysis. Figure 4 shows these words sized by the frequency of words within the document set.

Table 5: Top 30 words and frequencies generated with search phrase: Natural Language Processing

\begin{tabular}{|c|c|}
\hline Word & Number of Occurrences \\
\hline Cost Function & 260 \\
\hline Figure & 159 \\
\hline Morphological Analysis & 118 \\
\hline Empirical Cost Function & 114 \\
\hline NLP & 102 \\
\hline Proceedings & 101 \\
\hline ASP & 88 \\
\hline Function F & 79 \\
\hline Syntactic Analysis & 76 \\
\hline English & 75 \\
\hline $\mathrm{Eq}$ & 74 \\
\hline Language & 71 \\
\hline Function Node & 70 \\
\hline X Language & 58 \\
\hline Fig & 56 \\
\hline $\mathrm{Sec}$ & 54 \\
\hline Cost Function C & 52 \\
\hline Y Subject Language & 47 \\
\hline Sigmoid Function & 47 \\
\hline Lexical Analysis Graph & 46 \\
\hline Function Approximation & 44 \\
\hline Empirical Cost Function $\mathrm{C}$ & 42 \\
\hline Sentiment Analysis & 41 \\
\hline Semantic Analysis & 41 \\
\hline Morphological & 40 \\
\hline Activation Function & 39 \\
\hline Pair Subject Language Code & 37 \\
\hline Recursive Function & 36 \\
\hline Machine Learning & 35 \\
\hline Teller Machine & 34 \\
\hline
\end{tabular}




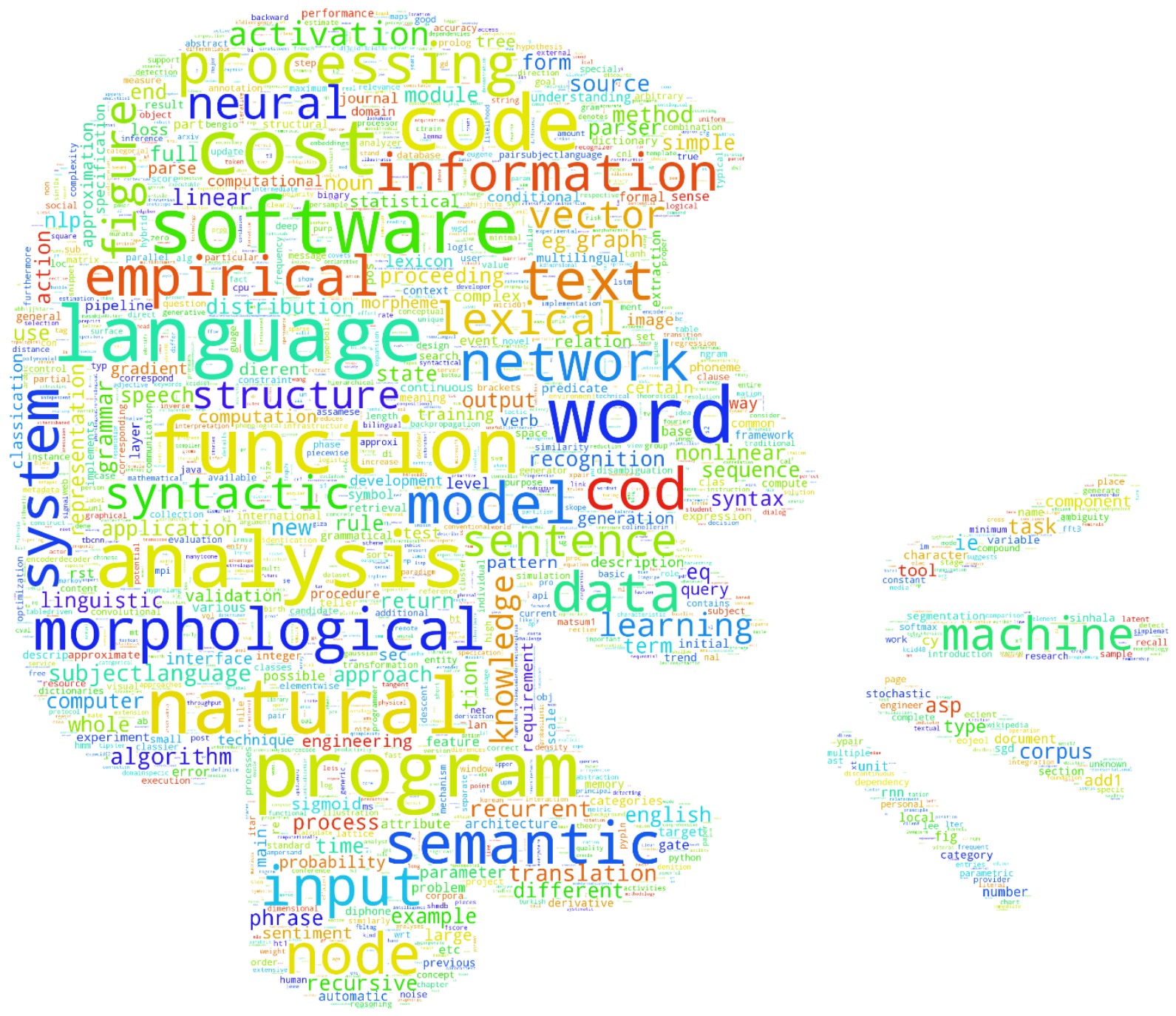

Figure 4: Output distribution word cloud of the search phrase: Natural Language Processing

\section{LIMITATIONS}

While conducting our research, we encountered some limitations of the project. We only used 100 articles for each scientific topic because of the computational limitations of the computers used. Each search varied in number of PDFs, but we ensured consistency in corpus size for each analysis. The data sets grew to around 600,000 strings and 29,000,000 characters after being parsed with n-grams. Although these strings and characters might seem large, the files are not inhibiting. However, iterating over each string can take some time. The program required around twenty minutes to run the corpus creation where we downloaded each PDF and extracted and filtered the text, then another half hour to run our analysis program. The PDF parser program we developed is somewhat inefficient. Most of the time the parser worked, however, when a PDF was older than a certain date, had too many pictures, or was too short, the text would emerge fused in 
a single string or in ASCII characters, forcing us to eliminate that document. In the future, we will seek more reliable means of extracting text from PDFs.

\section{CONCLUSION}

The results of our analysis demonstrate that we can evaluate trends of technology and methods in various disciplines. This information lays the groundwork for building a network of software used by various researchers to evaluate the effectiveness of National Science Foundation and other agencies' funding of different software projects. From these initial results, we are planning on continuing to improve the software to extract common methods and tools used in research in any given discipline from the literature, with the hope of connecting researchers to tools that they might not know about, or informing the development of future software packages to better address the needs of their users.

\section{REFERENCES}

Bird, Steven, Edward Loper and Ewan Klein (2009), Natural Language Processing with Python. O’Reilly Media Inc.

Hucka, M., \& Graham, M. J. (2016, May 8). ArXiv.org cs arXiv:1605.02265. Retrieved May 11, 2016, from https://arxiv.org/abs/1605.02265

Jones, Karen S. “Natural Language Processing: A Historical Review.” Language (2001): n. pag. Print.

"Natural Language Toolkit.” Natural Language Toolkit - NLTK 3.o Documentation. N.p., 9 Apr. 2016. Web. 15 Apr. 2016. <http://www.nltk.org/>.

Noam Chomsky's Theories on Language. (n.d.). Retrieved May 13, 2016, from $<$ http://study.com/academy/lesson/noam-chomsky-on-language-theories-lessonquiz.html>

Shinyama, Yusuke. "PDFminer." https://github.com/euske/pdfminer, 24 Mar. 2014. Web. 11 Jan. 2016. <https://github.com/euske/pdfminer>.

van Rossum, Guido. "Python Language." https://www.python.org/. Python Software Foundation, 20 Feb. 1991. Web. 19 Dec. 2015.

What is natural language processing (NLP)? - Definition from WhatIs.com. (n.d.). Retrieved May 13, 2016, from http://searchcontentmanagement.techtarget.com/definition/naturallanguage-processing-NLP 